CAOS 2017. 17th Annual Meeting of the International

\title{
Augmented Marker Tracking For Peri- Acetabular Osteotomy Surgery: A Cadaver Study
}

\author{
Silvio Pflugi ${ }^{1}$, Till Lerch ${ }^{2}$, Rakesh Vasireddy ${ }^{1}$, Nane Boemke ${ }^{3}$, Moritz Tannast ${ }^{2}$, \\ Timo M. Ecker ${ }^{2}$, Klaus Siebenrock ${ }^{2}$, Guoyan Zheng ${ }^{1}$ \\ ${ }^{1}$ Institute for Surgical Technology and Biomechanics, University of Bern, Bern, \\ Switzerland \\ ${ }^{2}$ Department of Orthopedic Surgery, Inselspital, University of Bern, Bern, \\ Switzerland \\ ${ }^{3}$ Institute for Anatomy, University of Bern, Bern, Switzerland
}

Silvio.pflugi@istb.unibe.ch, guoyan.zheng@istb.unibe.ch

\section{Introduction}

Peri-acetabular osteotomy (PAO) surgery is used for the treatment of adult hip dysplasia [1]. It is a demanding open surgery where the acetabular fragment is first detached from the rest of the pelvis and then reoriented to improve femoral head coverage. Many surgical navigation systems were previously introduced to assist the surgeon during a PAO surgery. The most widely used system nowadays is based on optical tracking. Dynamic Reference Bases (DRBs, active or passive) are attached to the patient's pelvis and fragment and are tracked by an external stereo-camera to measure the reorientation [2]-[4]. These systems offer high accuracy but suffer from drawbacks such as the line-of-sight impediment, high cost and a large setup in the operating room. Alternative techniques including electro-magnetic (EM) tracking which does not suffer from the line-of-sight impediment but is susceptible to magnetic field distortions [5], [6]. Hybrid navigation systems were proposed to overcome each individual technology's drawbacks but they are in general very complex and expensive [7]-[9]. In this study we validated a substantially smaller, easy to use and relatively cost-effective new hybrid navigation system where we directly place a tracking unit on the patient's pelvis to track an augmented marker attached to the acetabular fragment and compared it to a previously developed and validated optical trackingbased navigation system [4]. 


\section{Material And Methods}

A validation study including 3 pelvises (6 hip joints) undergoing navigated PAO was performed. Inclination and anteversion of two navigation systems for PAO were compared during acetabular reorientation. The hybrid system consists of a tracking unit which is placed on the patient's pelvis and an augmented marker which is attached to the patient's acetabular fragment (see Figure 1). The augmented marker is made up of a planar augmented reality marker [10] with an integrated Inertial Measurement Unit (IMU).
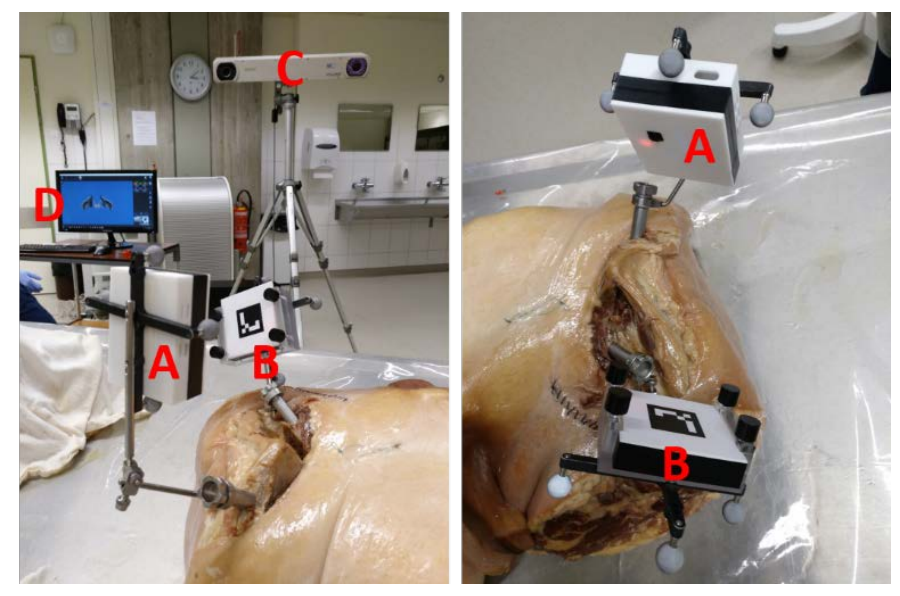

Figure 1: Left: Experimental setup: The tracking unit (A) attached to the pelvis and the augmented marker (B) attached to the acetabular fragment. Both are attached to DRBs for comparison to the optical tracking-based navigation system. The host computer (D) showing the 3D model of the pelvis is next to the stereo camera (C) in the back. Right: Close up view showing the tracking unit (A) and the augmented marker (B).

The tracking unit consists of a miniature computer and a camera module which sends a live video stream of the augmented marker to the host computer. Simultaneously, the augmented marker sends the orientation output from the IMU to the host computer. The host computer detects the marker in the video stream and estimates its pose using a robust pose estimation algorithm [11]. Anatomy registration is performed using a previously designed registration device [12] and solely relies on the IMU measurements to record the orientation of the patient's anterior pelvic plane (APP). During the reorientation procedure, the host computer uses the orientation from the augmented marker (if visible) or the orientation output from the IMU to update the acetabular orientation on the computer screen. For validation, we performed a cadaver study including 3 cadaver pelvises (6 hip joints). During acetabular reorientation, we reoriented the fragment to 20 random positions and simultaneously recorded inclination and anteversion from both systems. For each hip joint, we performed all necessary steps (anatomy registration and fragment reorientations) three times. This resulted in a total of 360 different reorientation positions.

\section{Results}

Mean absolute difference for inclination and anteversion $(\mathrm{N}=360)$ was 1.34 degrees and 1.21 degrees, respectively. The measurements from our system show a very strong correlation to the groundtruth optical tracking-based navigation system for both inclination and anteversion (0.9809 / 0.9711). 
A box plot showing the median error and the 5-th and 95-th percentiles for each individual case is shown in Figure 2.
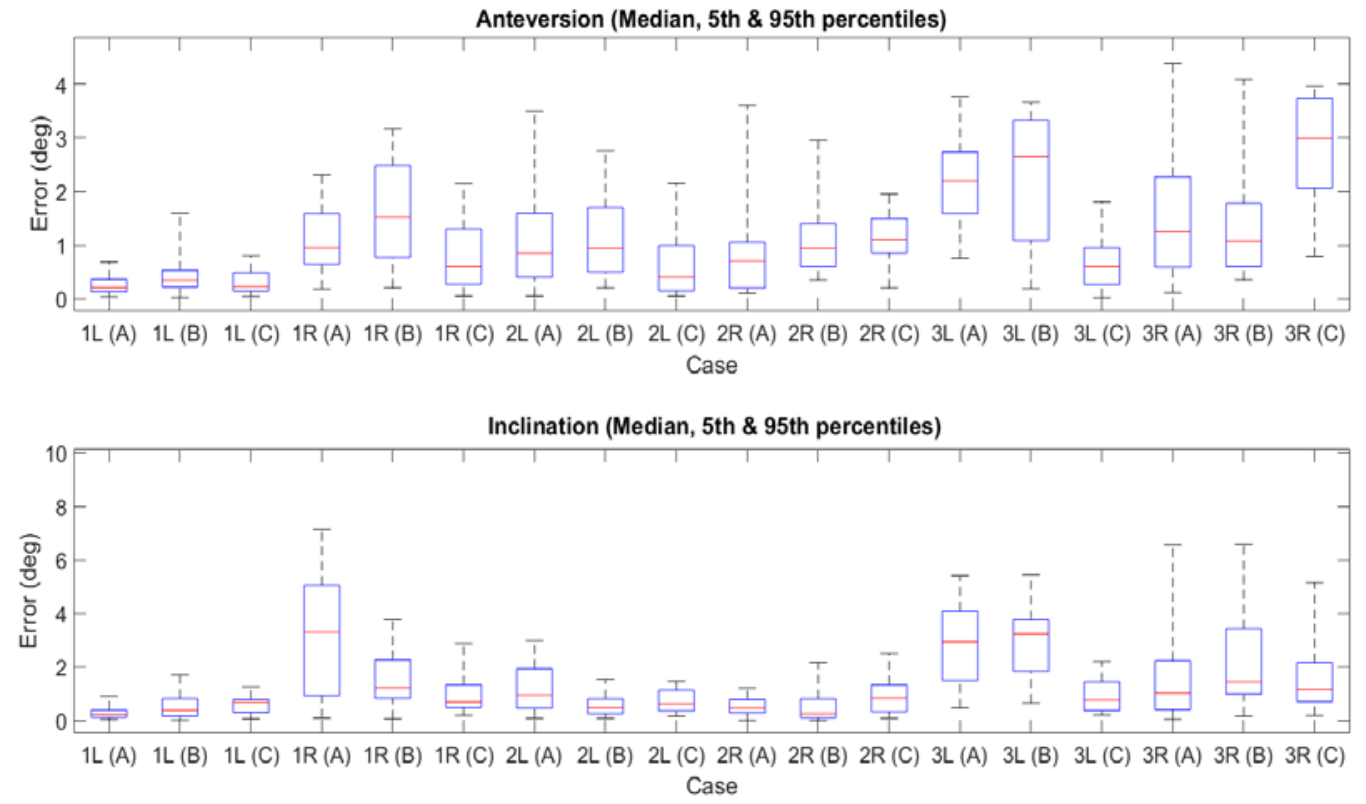

Figure 2: Error box plot for anteversion (top) and inclination (bottom). The 5-th and 95-th percentiles and the median error (red line) are shown for each case. Letters L/R represent hip joint side and letters A, B and $\mathrm{C}$ represent the first, second and third experiment for a specific hip joint.

\section{Discussion}

In this work, we successfully demonstrated the feasibility of our system to measure inclination and anteversion during acetabular reorientation. We compared the new system to a gold-standard optical tracking-based navigation system.

A strong correlation and minor mean differences for both anteversion and inclination were found. Advantages of this new system include minimal space requirements for the setup in the operating room and no line-of-sight impediment.

A limitation of this system is that it solely relies on the IMU during anatomy registration. IMUs offer a lower accuracy due to known issues such as drift [13], [14]. However, the time to perform the registration is very short, thus limiting effects such as drifting. During reorientation, drift is negligible since we fully rely on the marker tracking as long as it is visible and only rely on the IMU in times when the marker is not visible. This can be avoided with a careful setup in the beginning. Further, we only compare our system to the current gold standard and we did not perform an additional CT scan for direct evaluation. This means that error sources that are present in optical tracking-based navigation such as anatomy registration (soft-tissue between digitization pointer and bony landmark) are included in this study. 


\section{References}

[1] P. Hsieh, Y. Chang, and C. Shih, "Image-guided periacetabular osteotomy: computer-assisted navigation compared with the conventional technique: a randomized study of 36 patients followed for 2," Acta Orthop., 2006.

[2] M. Jaeger, B. Westhoff, A. Wild, and R. Krauspe, "Computer-assisted Periacetabular Triple Osteotomy for Treatment of Dysplasia of the Hip," Zeitschrift fuer Orthop. und Ihre Grenzgebiete, vol. 142, no. 1, pp. 51-9, 2004.

[3] F. Langlotz and M. Stucki, "The first twelve cases of computer assisted periacetabular osteotomy,” Comput. Aided ..., 1997.

[4] L. Liu, T. Ecker, and S. Schumann, "Computer assisted planning and navigation of periacetabular osteotomy with range of motion optimization,” Med. Image Comput. Comput. Interv. MICCAI 2014, vol. 8674, pp. 643-650, 2014.

[5] R. Von Jako, J. Carrino, and K. Yonemura, "Electromagnetic navigation for percutaneous guide-wire insertion: accuracy and efficiency compared to conventional fluoroscopic guidance," Neuroimage, 2009.

[6] H. Zhang, F. Banovac, and R. Lin, "Electromagnetic tracking for abdominal interventions in computer aided surgery," Comput. Aided ..., 2006.

[7] S. Beller and S. Eulenstein, "Upgrade of an optical navigation system with a permanent electromagnetic position control,” J. Hepatobiliary Pancreat Surg, vol. 16, no. 2, pp. 165-70, 2009.

[8] G. Claasen, P. Martin, and F. Picard, "High-bandwidth low-latency tracking using optical and inertial sensors,” Autom. Robot. Appl. (ICARA), 2011 5th Int. Conf., pp. 366-371, 2011.

[9] M. Haid, M. Kamil, T. Chobtrong, and E. Guenes, "Machine-vision-based and inertial-sensorsupported navigation system for minimal invasive surgery,” AMA Conf. 2013 - Sens. 2013, 2013.

[10] S. Garrido-Jurado, R. Munozoz-Salinas, F. J. Madrid-Cuevas, and M. J. Marin-Jimenez, "Automatic generation and detection of highly reliable fiducial markers under occlusion," Pattern Recognit., vol. 47, no. 6, pp. 2280-2292, 2014.

[11] G. Schweighofer and A. Pinz, "Robust pose estimation from a planar target," IEEE Trans. Pattern Anal. Mach. Intell., vol. 28, no. 12, pp. 2024-2030, 2006.

[12] S. Pflugi, L. Liu, T. Ecker, J. Cullmann, K. Siebenrock, and G. Zheng, “A cost-effective surgical navigation solution for periacetabular osteotomy (PAO) surgery,” Comput. Radiol. Orthop. Interv., pp. 333-348, 2016.

[13] K. O’Donovan and R. Kamnik, "An inertial and magnetic sensor based technique for joint angle measurement,” J. Biomech., 2007.

[14] A. Behrens and J. Grimm, "Inertial navigation system for bladder endoscopy,” 2011 Annu. Int. Conf. IEEE Eng. Med. Biol. Soc., pp. 5376-5379, 2011. 